\title{
The Clinical Assessment of Low Mood
}

Dr Ramya Srinivasan

Wellcome Trust Clinical Training Fellow/ST6 in Child and Adolescent Psychiatry

UCL Division of Psychiatry, 6th Floor - Maple House, 149 Tottenham Court Road, London W1T 7NF

UCL GOS Institute of Child Health, 30 Guilford Street, London, WC1N 1EH

Email: ramya.srinivasan.12@ucl.ac.uk

Dr Andrew McWilliams

Department of Child and Adolescent Psychiatry, Institute of Psychiatry, Psychology and Neuroscience, 16 de Crespigny Park, London SE5 8AF, UK.

Great Ormond Street Hospital for Children NHS Trust, Great Ormond Street, London WC1N 3JH, UK.

Metacognition Group, Wellcome Centre for Human Neuroimaging, Institute of Neurology, UCL, 12

Queen Square, London WC1N 3AR, UK

Email: andrew.mcwilliams@ucl.ac.uk

Prof David Skuse

Professor of Behavioural and Brain Sciences and Honorary Consultant in Developmental Neuropsychiatry

UCL GOS Institute of Child Health, 30 Guilford Street, London, WC1N 1EH

Email: d.skuse@ucl.ac.uk

Word count: 2273

\section{Introduction}

The prevalence of depression is increasing and it is considered to be the single largest contributor to global disability by the World Health Organisation (World Health Organization, 2017). Depression is more common in patients with physical illness: around $20 \%$ of people with a chronic physical health problem suffer from depression (Anderson, 2001; NICE, 2009) and this rises to around a third in 
medical inpatients. Depressive illness, even at low levels of severity is associated with higher rates of morbidity and mortality in those with physical illness (Rai et al., 2010; Meltzer et al., 2012).

Depression has been shown as an independent risk factor both for cardiovascular disease (Glassman and Shapiro, 1998) and for mortality associated with the cardiovascular disease (Frasure-Smith, Lesperance and Talajic, 1995). Higher rates of depression are also found in diabetes and metabolic syndrome, other autoimmune conditions, asthma, stroke, chronic pain syndrome and multiple sclerosis (Shelton and Miller, 2010; Slavich and Irwin, 2014). Cancer has been robustly associated with increased rates of depression, which affects up to $20 \%$ of patients (Mitchell et al., 2011). There is increasing evidence that inflammation plays a key role in the pathogenesis of depression for a subset of individuals (Kiecolt-Glaser, Derry and Fagundes, 2015). Thus, there may be a shared underlying aetiology between depression and some chronic physical illnesses. Low mood can also be a key symptom in some medical illnesses (such as hypothyroidism and Vitamin B12 deficiency). In addition, low mood is an iatrogenic side-effect of many treatments (steroids, hormonal treatments such as androgen deprivation therapy).

Depression will often affect an individual's ability to engage with their healthcare for physical problems. Low motivation, self-esteem and self-confidence may all affect attendance at and engagement with medical appointments, taking medication consistently and participation in physical activity or rehabilitation programmes. It is important that doctors remember that although depression is likely in physical illness, it is not a necessary sequelae of having a serious or chronic physical health problem. Thus its presence, although expected, should not be dismissed as unavoidable and hence not needing management. Depression has a significant impact on quality of life and long-term outcomes. It is important to identify and treat in a timely fashion and all doctors should have some skills in doing this. 
The term 'mood disorder' refers to conditions in which the main feature is an abnormality of mood. Most commonly this is low mood as in depression but elevated mood can be seen in mania. This article aims to provide an approach to assessing low mood in patients in the general hospital setting.

\section{What is depression?}

The aetiology of mood disorders is not fully understood; the cause is likely to be multifactorial involving complex interplay between genetic factors and life experience. Stressful life events such as those relating to physical health may trigger the disorder.

Depression is a heterogeneous disorder with varied clinical presentations. The core symptoms in current classification systems are low mood, anhedonia (loss of ability to experience pleasure) and reduced energy levels. Other symptoms include: disturbed sleep, disturbed appetite, poor concentration, agitation or slowing of movements, low self-confidence, feelings of guilt or shame, and suicidal thoughts or acts. The ICD-10 (World Health Organization, 1992) requires at least four depressive symptoms present for at least two weeks, with each symptom present for most of every day. At least two of these must be core symptoms. The ICD-10 determines severity of depression by the number of symptoms present, and clinicians will consider the intensity of symptoms of and the level to which functioning is affect when making a judgement about severity. In cases of severe depression, delusions and hallucinations can be experienced. These should usually have content in keeping with the person's low mood (be "mood congruent"), such as a person thinking they are in fact dead or that everyone can smell their faeces. If such symptoms are experienced the disorder is referred to as psychotic depression. Mood incongruent symptoms should prompt consideration of a different psychotic diagnosis, not depression.

It is important to note that low mood may not be the most prominent feature in a given individual; for example, in some people, irritability may be more obvious than the core features themselves. 
Other symptoms are sometimes categorised (see Box 1) as 'biological', 'psychological' (such as feelings of guilt, worthlessness, reduced self-esteem, pessimism, and ideas of self-harm or suicide) and 'cognitive' (poor concentration, poor memory). Frequently these symptoms can therefore imitate dementia, when it is referred to as depressive pseudo-dementia and presents a potential diagnostic challenge (see Box 2. Vignette 1). It is also important to consider common differential diagnoses. These include adjustment disorder where depressive symptoms, often in the context of a life event including acute illness, are present but, the severity or time criterion for a depressive disorder are not met, hypoactive delirium where a patient may present as flat and unresponsive but is in fact acutely confused, and bereavement reaction where depressive symptoms may be present in the context of bereavement.

There are different rating scales that can be used by clinicians to screen for and assess the severity of depression. Scales (see Box 3) that are useful in General Practice and general hospital settings include the Patient Health Questionnaire (PHQ-9) (Kroenke, Spitzer and Williams, 2001) and Hospital Anxiety and Depression scale (HADS) (Zigmond and Snaith, 1983). It is important to note that these instruments should be used to as a starting point for sensitive discussion and further exploration of a person's mental health with a clinician, rather than to diagnose depression. In particular, they do not fully cover aspects of risk or the full range of possible depressive symptoms. See Box 4 for examples of questions that may be useful in exploring depressive symptomatology.

Patients with depression may present with additional physical symptoms or may be overly anxious about their physical health. If this occurs on top of pre-existing physical illness, symptoms may be out of proportion to the underlying pathology, but making a confident judgment about the relative contribution of physical illness and psychological factors is very tricky. Identifying depression will be more difficult, especially as the 'biological' symptoms of depression are also very common in many different physical illnesses. Depression is commonly associated with anxiety which can also present with physical symptoms. Furthermore, it is important to note that even sub-threshold depressive 
symptoms may be significantly disabling when occurring in conjunction with physical health problems (Rai et al., 2010).

In mild depression, NICE guidelines recommend psychological talking therapies rather than antidepressant treatment in the first instance (NICE, 2009, 2012; NCCMH, 2010). Anti-depressants should be considered in those with moderate or severe depression, in mild depression complicated by significant physical health problems, and in sub-threshold or mild symptoms which have been present for two years or persisted despite other interventions (NICE, 2009, 2012; NCCMH, 2010).

\section{History}

All medical specialities use history and examination to formulate a diagnosis and psychiatry is no different. It is particularly important to obtain collateral information from someone who knows the patient, as they themselves may be unable or unwilling to describe their difficulties sufficiently.

When assessing low mood, the history should cover the nature of the symptoms of depression, their onset, duration, severity and impact on functioning. In the general hospital setting, close attention to the timing of low mood and the course of a physical illness (e.g. in relation to receiving a diagnosis) can be useful. A wide range of psycho-social factors are important in the development, diagnosis and prognosis of depression which should be explored whilst bearing in mind a biopsychosocial model (Box 5). It is important to bear in mind that social adversity is a significant factor which may contribute both to depression and to poor physical health outcomes (see Box 2 . Vignettes 2 and 3)

Important aspects of history to cover include:

- Past Psychiatric History - has the person ever received any previous psychiatric diagnosis, details of these, including who made them, lifetime history of low mood, any treatment received (whether 
from GP or mental health services), mental health admissions and history of risk to self and others. Collateral information is particularly important to corroborate this and ideally should be established via communication with the professionals who were managing the patient at the time. Depressed people may not able to give an accurate account of their past treatment.

- Medical and Surgical History - explore any ongoing health concerns or new diagnosis and their impact on the person's life and mood. (See Box 2. Vignettes 4 and 5)

- Family History - with focus on the family constitution and presence of diagnosed mental health disorders.

- Developmental History - covering birth and childhood development and education. Questions about current domestic violence should not be missed as this may the person's only chance opportunity to seek help. Probing questions about long past childhood abuse are often of limited use in a general medical setting if the patient does not volunteer this spontaneously, and may in fact cause distress of jeopardise the relationship with the clinician.

- Social History - covering accommodation, past and current relationships, social support, employment, finances and housing.

- Substance and Alcohol use - both current and historic: what is being used and how often, has this changed recently, what effect it has on the person's mood and functioning. It may also be important to consider the impact on physical health.

\section{Mental State Examination}

The mental state examination is the systematic examination and summary of specific aspects of a person's mental experiences and behaviour at the time of assessment; it can be used to provide snapshot of a person's mental world at a specific point in time or can be assembled as a view over a brief period, such as the last two weeks. The latter approach means that moment-to-moment or 
daily fluctuations do not obscure the broader picture of recent mental experience. Together with the history, this information allows diagnosis of mental disorder and its severity to be assessed. This is an approach taken by psychiatrists and clinical assessment in the general hospital by general hospital clinicians may be more pragmatic. Described below are possible findings to consider in depression.

Appearance and Behaviour - Clothes and grooming may be neglected (ill-fitting, old and shabby or dirty). The eye-line may be down or avoidant, with furrowing of the forehead, down-turning mouth and hunched shoulders; however, it is important to remember that some people may experience feelings of depression whilst superficially appearing cheerful. Psychomotor retardation (the slowing of movements due to a psychiatric cause) may be seen. Some people exhibit psychomotor agitation, with restlessness and over-activity, such as wringing hands or even pacing, and this is more common in the middle-aged and elderly.

Speech -This may be quiet, slow and monotonous, with a lack of spontaneity. You may feel that you have to push to get answers by asking numerous closed questions as the person is not volunteering information.

Mood - It is usual practice to note the person's subjective account of their mood, either by describing their view or by quoting directly the actual words they use to describe it. They may feel low or irritable or even anxious, and describe as it being qualitatively different from more ordinary sadness in some way. Traditionally, the assessor also makes an objective observation of the person's mood which may be at odds with the subjective account. Core symptoms of depression should be enquired about such as low energy and anhedonia. 'Biological', 'cognitive' and 'psychological' (Box 1) symptoms should also be explored. It is also crucial to conduct a risk assessment (see Box 6), asking directly about suicidal ideation, deliberate self-harm, self-neglect and vulnerability.

Thoughts and Perceptions - The person may be preoccupied by depressive cognitions, and in psychotic depression the person may present with delusions along these themes. For example, a personal with delusional guilt may falsely believe that they have committed a serious crime. 
Delusions relating to physical ill-health, financial difficulties and persecution may be reported. In severe cases, people may hold extreme nihilistic beliefs that they have lost body parts, are rotting or are even dead.

Cognition - Examination it usually accomplished using standardised screening tools, such as the MMSE (Folstein, Folstein and McHugh, 1975), the ACE-III (Noone, 2015), the MOCA (Nasreddine et al., 2005) or the Frontal Assessment Battery (Dubois et al., 2000). Depression may produce deficits that mimic a range of dementias, but people may also seem to have low motivation to engage with testing and this clinical observation is in itself informative diagnostically.

Insight - This refers to whether a person shares the clinician's views of the low mood, its causes and any treatments. It should not simply be considered as present or absent but as existing along a continuum. Poor insight makes treatment challenging for clinician and patient but addressing this may well form a focus for that treatment. In depressive disorders of mild or moderate severity, some degree of insight is usually retained; however, a person may not be fully aware of the effect their mood may be having on their daily functioning or physical health. In severe cases or where psychotic symptoms are present, insight may be lost almost completely.

Once the history and examination are complete these are synthesised together into a biopsychosocial formulation (Box 4) of the patient's presentation, including a description of the risks they pose to themselves, to others and from others. This is a method used by psychiatrists to contextualise a patient's experience and to refine management options; a more pragmatic approach may be required in the general hospital setting but the principles may be of benefit when considering management (See Box 2 for vignettes of presentations and potential issues to consider). Risk assessment (Box 6), especially related to suicidal ideation, will not be discussed in detail here as it is important but complex and specialist support should be sought if in any doubt. 


\section{Moving towards management}

Resources to support and treat people with depression may be accessed via the GP, local psychological services based with primary care or secondary mental health services. It is important to consider whether you are the most appropriate person to make decisions about low mood and to consider whether liaison psychiatry or a ward psychology input may be required if available locally. The nature of this discussion and the decision made would most likely depend on the individual patient's presentation and level of risk; for those about whom there are serious concerns urgent psychiatric input should be sought. With regard to management, there is good evidence for nonpharmacological approach including psychological therapies in mild-moderate depression whilst antidepressant medication may be required for moderate-severe depression (NICE, 2009, 2012; $\mathrm{NCCMH}, 2010)$. It is also important to bear in mind that most cases of depression are managed in general practice (NICE, 2004). Within the general hospital setting, ensuring adequate pain control, encouraging visits from friends and family, engagement with activities and assisting with sleep may help alleviate symptoms.

\section{Conclusion}

Depression is a common disorder and can present in many different ways. Despite the intuition that it is easy to detect depression as it is common and close to the realm of many doctors' own personal experience, assessment does require planning and sensitivity, along with knowledge of the condition and recognition of heterogeneity between patients. Given the prevalence of depression and its interaction with physical health problems, all doctors need some understanding of how to assess mood and how to refer on where necessary. 
Funding

Andrew McWilliams is a National Institute for Health Research-funded academic clinical fellow.

Ramya Srinivasan is a Wellcome Trust Clinical Research Fellow and was previously and National Institute for Health Research funded academic clinical fellow. 


\section{Key Points}

- Depression is more common in those with chronic physical health problems and is associated with increased risk of morbidity and mortality. All doctors therefore need some understanding of how to identify those who may be experiencing depression.

- The assessment of depression involves taking a history of both psychological and social factors, and conducting a mental state examination with a particular focus on mood.

- Depression is characterised by 3 core features plus a number of additional symptoms. The core features are: low mood, a loss of enjoyment of previously pleasurable activities and reduced energy levels.

- Other symptoms are sometimes categorised as 'biological ', 'cognitive' and 'psychological' and this can be a useful structure for questioning approaches. Biological and cognitive symptoms may be difficult to disentangle from the symptoms of chronic physical health problems.

- It is important to assess risk to self and others. This includes not only suicidal ideation and deliberate self-harm, but also self-neglect as a risk to physical health. Risk to children should also be considered, especially if any are dependent on the patient. 


\section{Box 1 - Biological, Cognitive and Psychological symptoms of depression}

'Biological symptoms' - include the disturbance of sleep, appetite, libido and diurnal variation in mood. Sleep is most often described as being reduced - there may be delay of sleep onset, though waking early (more than 2 hours before the alarm clock) is characteristic of depression of increasing severity. In some cases excessive but unrefreshing sleep may be reported. Appetite may be diminished, leading to weight loss, but some patients may have increased appetite. Loss of sexual libido is important to consider at initial assessment, though as it is a common side-effect of many medications including selective serotonin re-uptake inhibitors (SSRIs). Diurnal variation in mood is the phenomenon of mood being worse in the morning and then gradually improving throughout the day. Although mood maybe better in the evening it will remain low.

'Cognitive Symptoms' - the cognitive symptoms of depression tend to receive less attention than the other symptoms but are common and are often debilitating. They may be confused with other conditions. They include difficulty concentrating, distractibility, forgetfulness and poor memory. It is therefore important to test cognition formally, especially in the elderly where depression may present as hypo-active delirium or as pseudo-dementia. In the latter, reported cognitive deficits are regarded as secondary to the mood disorder itself and so generally recover once the depressive diagnosis has been treated.

'Psychological symptoms' - relate to the emotional content of thoughts connected with low mood such as such as feelings of guilt, worthlessness, reduced self-esteem, social worries, anxiety about health and negativity about the future. 


\section{Box 2 - Vignettes}

\section{An 82-year old man has been brought in by his care home for an orthopaedic procedure to his}

foot. He seems a little withdrawn and is slow to answer questions, which one of the junior doctors finds this a little irritating as she thinks he is putting it on.

- Depression is very common in the elderly and may multifactorial, with links to feeling a loss of a role in society, declining physical function, isolation and reflections on mortality, as well as the burden of chronic disease and medication side-effects. Could there be an alternative diagnosis?

- Dementia commonly presents in a similar manner to depression. Vigilance for and early detection of dementia will hence allow accurate diagnosis.

- Hypoactive delirium is often missed in acute hospitals and may have been present in the care home prior to admission.

- Treatments for depression, which may co-occur with either of the above, might include a careful psycho-social support package and talking therapy. Anti-depressants would need to be introduced slowly and with care to monitor for life-threatening autonomic side-effects such as hypotension.

- It is not uncommon for health professionals to feel irritated or frustrated by patients, particularly those who do not seem to be improving as they are expected or who take up time during a busy shift. Frustration at events or at work pressures can sometimes be misdirected towards patients, and sometimes the experiences of the patient themselves (if they are feeling worthless or like they are wasting a doctor's time) can affect the way we feel about them. It is important to be mindful of these feelings and think about where they come from, so that they don't get in the way of the care patients receive. 


\section{A 41-year old woman was been admitted to your medical ward two days ago as a result of}

asthma. During a ward round she discloses that she lost her job 4 weeks ago and she keeps crying about little things.

- Consider whether you can come back later on your own to discuss this in more detail before deciding if you might need support from your liaison psychiatry team.

- You need to set aside some time and find a private space to talk if possible: drawing the curtains around the bed can only generate an illusion of privacy.

- Do some research first: is there a record of mental illness or medications from primary care record? - You need to ask about the diagnostic features of depression but eliciting these in requires a flexible approach if the information you generate is going to be meaningful. Demonstrating that you view the patient's predicament as common can encourage the patient to share: "Lots of people might find your situation difficult to cope with."

- Then screen for the core features of depression - low mood, lack of energy and anhedonia

- Further questions could explore biological features (sleep, appetite), cognitive features (concentration, memory) and psychological features (self-esteem, worthlessness, plans for the future)

- Direct questions about suicidality now and in the past are often answered in a surprisingly straightforward manner. Think about self-neglect - have recent difficulties affect her ability to manage her asthma?

- Consider how her current circumstances fit into her presentation - e.g. did she lose her job because of her depressive symptoms, or start to feel depressed after losing her job?

- Think about whether she uses alcohol or drugs to excess.

- Have a low threshold for involving a psychiatrist. 


\section{A 64-year old man with type 2 diabetes mellitus, who is well-known to you, comes to your}

diabetes clinic for his annual follow-up. You notice recent poor control of his diabetes which is unusual for him. He breaks down into tears in front of you, saying his wife died suddenly 3 months ago. He has no history of mental illness known to your team.

- Is this man experiencing a normal grieving reaction or is he depressed? The prevalence of depression older adults is high, but it is important not to pathologise normal grieving. There is dispute between psychiatrists about the length of time that normal grieving should take, so the duration may be a less useful indicator of whether this man is depressed than establishing the nature of his depressive symptoms. How pervasive are his sad feelings through the day? Is he feeling hopeless or suicidal?

- Consider whether you need collateral history to be confident your screening is accurate. Obtaining this is a chance to understand his informal support networks.

- Does this man need further support? Are you the best person to make this decision? Psychiatry or psychology services may not be available to you in a timely fashion. However, the patient's GP may know him very well and often will be able to see him more quickly than a specialist service. There is an element of judgement in this as the patient may decide not to follow through on a GP appointment. If you are concerned about this possibility it might be worth involving a relative or friend to encourage him to attend or asking the GP directly to assertively arrange a follow up appointment for him.

- Think about the management of his diabetes in the context of recent events - will he need additional help with this?

- Seek advice about support services available, but consider specialist 3rd sector organisations for bereavement, such as Cruse in the UK. 


\section{35-year old woman with breast cancer says during chemo that she doesn't "feel like a woman}

any more" and she "is not sure if it's worth going on with life."

- She has told you thoughts which sound suicidal and she needs a fuller assessment of these. You need to ensure that an appropriate professional will be seeing her in a timely manner. Many oncology services will have a dedicated psychologist in the clinic, who may be the best person to explore her history and obtain collateral accounts that day.

- This woman is at a high risk of developing depression secondarily to her breast cancer and treatment. Chronic illnesses can induce direct physiological effects on the brain associated with depression, as can the medications we prescribe.

- The psychological effects of a life-threatening illness are obviously profound, she may instead be grieving the roles she is losing within her family, employment and society. A previously fit woman may struggle to realise she cannot control her own health and she may consequently feel apathetic towards her body. Coping with such changes can be difficult and this could be understood as an adjustment reaction.

- latrogenic effects of treatment contribute to all these problems. Additional challenges include adjustment to changes in physical appearance, including loss of hair, surgical scars (whether visible or hidden), fertility impairment and the array effects of steroids have on the body. These may make her feel marked out as ill feel less sexually attractive and unable to maintain her previous social roles.

- Treatment for depression may be appropriate, via medication or talking therapy. Even if the cancer remits she may remain fearful of recurrence. 


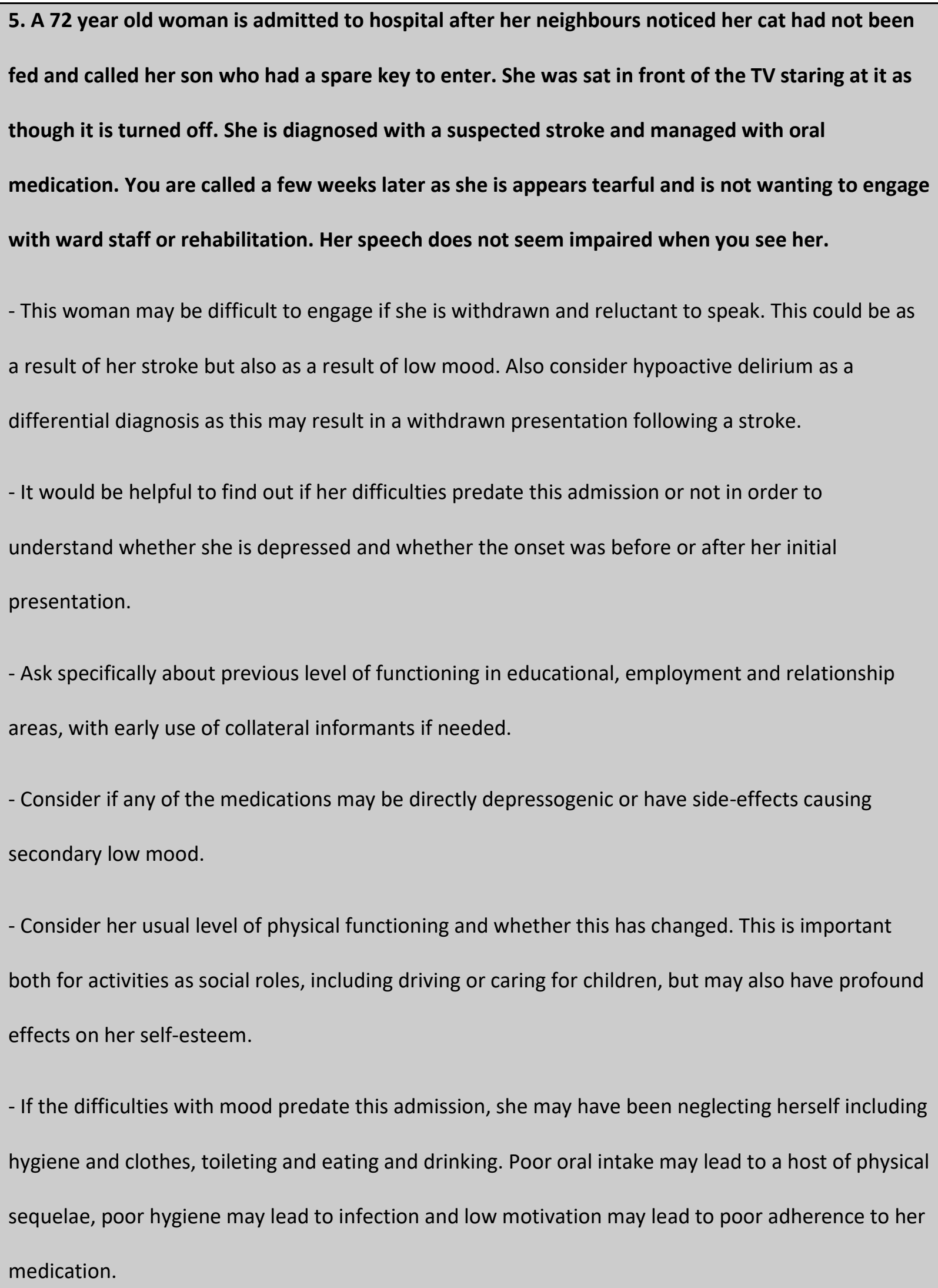

- If the difficulties with mood predate this admission, she may have been neglecting herself including hygiene and clothes, toileting and eating and drinking. Poor oral intake may lead to a host of physical sequelae, poor hygiene may lead to infection and low motivation may lead to poor adherence to her medication. 
- It will be difficult to address her physical health difficulties unless her low mood is also addressed.

Stroke commonly brings about other changes in mood, such as irritability or a lability of mood.

Personality changes may be prominent and affect how the person relates to friends and relatives.

- If she needs to take medication long term, consider exploring her feelings around this. Many people disengage with medical plans and as they do not want to feel medicalised or because daily tablets may force them to confront their mortality more frequently than they might hope for. This episode may precede vascular dementia and assessment for dementia should be considered again.

- It will be important to consider offering her a structured rehabilitation programme, counselling if necessary and to consider medication for depression. Occupational therapist assessment of the home may allow the person to remain independent though supply of adjustments to the house can be felt as stigmatising and as signifying illness and hence experiences negatively. Support can be offered via stroke charities (such as the Stroke Association in the UK) which offer helplines and coordinate resources to support carers. 


\section{Box 3 - Rating Scales}

\section{Hospital Anxiety and Depression (HAD) Scale:}

- Developed to assess anxiety and depression in a general medical population

- There are 14 self-report items in total, with 7 questions on depression and 7 on anxiety.

- Useful in those with physical health problems as focuses on psychological and cognitive symptoms of depression, rather than the biological symptoms which may be difficult to disentangle from physical health difficulties but does NOT include all the diagnostic criteria as a result

- Does NOT include any questions on risk

- Can also be used in primary care

\section{Patient Health Questionnaire (PHQ-9):}

- A 9-item, self-report questionnaire, initially developed to assess severity of depression

- Validated for use in primary care and can be used as an initial screening measure in at-risk populations e.g. those with coronary heart disease or stroke

- Is based on the diagnostic criteria for major depressive disorder in the (now superseded, though still useful) Diagnostic and Statistical Manual - Fourth Edition (DSM-IV) but would still require followup questions in order to assess fully symptomatology

- Does ask about suicidality but not to the level sufficient to constitute a meaningful risk assessment if an individual answers positively 
Box 4 - Eliciting the symptoms of depression: suggested questioning approaches

Low mood:

How have you been feeling in yourself recently?

How has your mood been lately?

Are you managing to keep your spirits up, or are things getting on top of you?

Does your mood vary over the course of the day?

If you had to rate your mood out of ten, with 0 being the worst you've ever felt, 10 being the best and 5 being somewhere in the middle, how would you say it has been over the past couple of weeks?

Anhedonia:

What kind of things do/did you enjoy? Do you still enjoy these things?

Have you lost enjoyment in things you used to enjoy?

Are there things you enjoy doing at the moment, such as hobbies or seeing friends?

Reduced energy and activity levels:

How are your energy levels?

How do you spend your day?

Are you getting out and about still?

Everybody gets tired sometimes, but are you finding your energy levels are really low at the moment? 
Depressive Cognitions:

How confident do you feel in yourself?

Do you ever feel that you're not as good as other people or even that you are worthless?

Do you ever feel that you've done anything wrong?

Do you feel guilty about anything?

How do you see the future?

Do you ever feel hopeless about yourself or your life?

\section{Suicidal ideation and Self-harm}

Questions about suicide or self-harm often follow on naturally from those about depressive cognitions and may make it easier to ask these questions. There is no evidence to suggest that asking about suicidal ideation or self-harm increases a person's risk, and often people find it a relief to be able to be given the opportunity to talk about these topics.

Sometimes, people who feel low may feel that life feels too hard, have you ever felt that way?

Are you feeling that life isn't worth living?

Does life ever feel like a burden?

Have you ever had thoughts of harming yourself or ending your life? Have you thought about how you might do this?

Do you think you would do something to harm yourself or end your life?

Have you ever tried? Tell me a bit more about that. 


\section{Box 5 - Biopsychosocial model of formulation}

Developing a formulation identifying the biopsychosocial factors and how they contribute to an individual's presentation can be helpful in developing a coherent management plan. The biopsychosocial model of formulation was developed by George Engel who believed that 'all three levels, biological, psychological, and social, must be taken into account in every health care task.' These can be thought of as follows:

Biological factors - depression can make an individual more susceptible to developing a physical illness and vice versa. Individuals with depression can have deranged endocrine, immune, and neurotransmitter system functioning. Genetic factors such as family history as well as pre-existing physical and neurological problems are also included.

Psychological factors e.g. negative patterns of thinking, difficulties in coping, impaired emotional intelligence.

Social factors e.g. traumatic situations, early separation, family conflict, financial/work difficulties, lack of social support, bullying/harassment.

The above factors may be further delineated according to time in relation to the current episode as predisposing, precipitating, perpetuating and protective.

For example, if an individual is depressed with predisposing negative thinking styles, precipitating physical ill-health and perpetuation work-related stress but with a protective factor of a supportive social network, a psychological intervention in combination with support to resolve the work difficulties and encouragement to engage with a supportive social network may be an effective initial treatment option for that individual. 


\section{Box 6 - Risk assessment}

It is crucial to screen for suicidal ideation, deliberate self-harm and self-neglect. This important topic is can only be considered in highly a condensed format here and thus this should not be regarded as anything more than a brief introduction.

\section{Suicidal Ideation}

Many people with depression describe passive thoughts of no longer wanting to be alive; for example, saying that they sometimes wished they wouldn't wake up the next day. As depression increases in severity these thoughts may become more frequent and intense, developing into clear intent to self-harm with well-formed, specific plans of how to do this.

\section{Self-neglect and vulnerability}

Neglect of personal care and surroundings may have serious consequences. Poor hygiene, poor oral intake and poor management of finances can all have physical health consequences and those with pre-existing illnesses may neglect to take prescribed medication regularly. Self-neglect may also make a person vulnerable to exploitation and other crimes from strangers or people they know.

\section{Harm to others}

This may be important to consider in certain circumstances; for example, in the case of a person who feels that the future is hopeless and extends this to the lives of family members/children. 


\section{References}

Anderson, R. J. (2001) 'The prevalence of comorbid depression in adults with diabetes: a metaanalysis ', Diabetes Care, 24(6), pp. 10069-11978.

Dubois, B. et al. (2000) 'The FAB', Neurology, 55(11), pp. 1621-1626. doi: 10.1212/WNL.57.3.565.

Folstein, M. F., Folstein, S. E. and McHugh, P. R. (1975) “"Mini-mental state”: A practical method for grading the cognitive state of patients for the clinician', Journal of Psychiatric Research, 12(3), pp. 189-198.

Frasure-Smith, N., Lesperance, F. and Talajic, M. (1995) 'Depression and 18-month prognosis after myocardial infarction', Circulation. 1995/02/15, 91(4), pp. 999-1005.

Glassman, A. H. and Shapiro, P. A. (1998) 'Depression and the course of coronary artery disease', Am J Psychiatry. 1998/01/20, 155(1), pp. 4-11.

Kiecolt-Glaser, J. K., Derry, H. M. and Fagundes, C. P. (2015) 'Inflammation: Depression fans the flames and feasts on the heat', American Journal of Psychiatry, 172(11), pp. 1075-1091. doi: 10.1176/appi.ajp.2015.15020152.

Kroenke, K., Spitzer, R. L. and Williams, J. B. W. (2001) 'The PHQ-9: Validity of a brief depression severity measure', Journal of General Internal Medicine, 16(9), pp. 606-613. doi: 10.1046/j.15251497.2001.016009606.x.

Meltzer, H. et al. (2012) 'Physical ill health, disability, dependence and depression: results from the 2007 national survey of psychiatric morbidity among adults in England', Disabil Health J. 2012/03/21, 5(2), pp. 102-110. doi: 10.1016/j.dhjo.2012.02.001.

Mitchell, A. J. et al. (2011) 'Prevalence of depression, anxiety, and adjustment disorder in oncological, haematological, and palliative-care settings: A meta-analysis of 94 interview-based studies', The Lancet Oncology, 12(2), pp. 160-174. doi: 10.1016/S1470-2045(11)70002-X.

Nasreddine, Z. S. et al. (2005) 'The Montreal Cognitive Assessment, MoCA: a brief screening tool for mild cognitive impairment', J Am Geriatr Soc, 53(4), pp. 695-699. doi: 10.1111/j.1532-

5415.2005.53221.x.

NCCMH (2010) 'Depression in adults with a chronic physical health problem: the NICE guideline on treatment and management ', RCPsych Publications.

NICE (2004) Management of depression in primary and secondary care: Clinical Guidelines 23.

NICE (2009) 'Depression in adults with a chronic physical health problem: treatment and management. NICE clinical guideline 91.'.

NICE (2012) 'Depression in adults with a chronic physical health problem: Evidence update March 2012. Evidence update 12.'.

Noone, P. (2015) 'Addenbrooke's cognitive examination-III', Occupational Medicine, 65(5), pp. 418420. doi: 10.1093/occmed/kqv041.

Organization, W. H. (1992) The ICD-10 Classification of Mental and Behavioural Disorders: Clinical Descriptions and Diagnostic Guidelines, World Health Organization. World Health Organization.

Rai, D. et al. (2010) 'Common Mental Health Disorders, subthreshold symptoms and disability: longitudinal study', The British Journal of Psychiatry, 197(5), pp. 411-412.

Shelton, R. C. and Miller, A. H. (2010) 'Eating ourselves to death (and despair): The contribution of adiposity and inflammation to depression', Progress in Neurobiology. Pergamon, 91(4), pp. 275-299. 
doi: 10.1016/J.PNEUROBIO.2010.04.004.

Slavich, G. M. and Irwin, M. R. (2014) 'Social signal transduction theory of depression', Psychiatric Bulletin, 140(3), pp. 774-815. doi: 10.1037/a0035302.From.

World Health Organization (2017) 'Depression and other common mental disorders: global health estimates', World Health Organization, pp. 1-24. doi: CC BY-NC-SA 3.0 IGO.

Zigmond, A. S. and Snaith, R. P. (1983) 'The Hospital Anxiety and Depression Scale', Acta Psychiatrica Scandinavica, 67(6), pp. 361-370. doi: 10.1111/j.1600-0447.1983.tb09716.x. 